\title{
Purposeful Model Parameters Genesis in Multi-population Genetic Algorithm
}

\section{Tania Pencheva* and Maria Angelova}

Unstitute of Biophysics and Biomedical Engineering - Bulgarian Academy of Sciences, 105 Acad. Georgi Bonchev Str., 1113 Sofia, Bulgaria

\begin{abstract}
IIn this paper recently proposed procedure for purposeful model parameters genesis, originally developed for simple genetic algorithm, has been validated for multi-population genetic algorithm when it is applied for the purposes of parameter identification of S. cerevisiae fed-batch cultivation. Proposed procedure aims to improve the algorithm effectiveness in respect to the convergence time and model accuracy treating intervals of variations of model parameters. Obtained results after the procedure application show more than $12 \%$ improvement of multi-population genetic algorithm convergence time while saving and even slightly meliorating the model accuracy.
\end{abstract}

Keywords: Multi-population genetic algorithm; Purposeful genesis; Model parameters; Fermentation process; Saccharomyces cerevisiae

\section{Introduction}

Wide application of fermentation processes (FP) in different branches of industry maintains contemporary scientific interest to investigate in this area. FP combine the dynamic of two fundamental components - non-biological and biological, but their specific peculiarities are ultimately determined from characteristics of live microorganisms. As complex, dynamic systems with interdependent and time-varying process variables, FP constitute a serious challenge for investigators working in the field of their modeling, optimization and control. Adequate modeling of FP mostly depends on the choice of a certain optimization procedure for model parameters identification. Genetic algorithms (GA) [1] are a good alternative to conventional optimization methods for solving such a complex problem [2-4].

The effectiveness of a certain optimization technique can be evaluated by the model accuracy achieved and the convergence time needed. Obtained promising results when purposeful model parameter genesis was originally developed and successfully applied for simple genetic algorithm [5], provokes the idea such a procedure to be tested for another kind of genetic algorithm. Thus, the aim of the study is to apply and validate a procedure for purposeful genesis for parameter identification of $S$. cerevisiae fed-batch cultivation when using multipopulation genetic algorithm.

\section{Materials and Methods}

\section{Multi-population genetic algorithms}

Standard genetic algorithms, originally presented in [1], search a global optimal solution using three main genetic operators in a sequence selection, crossover and mutation over the individuals in the population. While simple genetic algorithms work over one population at time, multi-population genetic algorithms (MpGA) is more similar to the nature since in it many populations, called subpopulations, evolve independently from each other. After a certain number of generations, a part of individuals migrates between the subpopulations.

\section{Procedure for purposeful model parameters genesis}

Due to the stochastic nature of genetic algorithms, a great number of algorithm runs have to be executed in order to obtain reliable results in parameter identification of a fermentation process model. When results from many algorithms executions were accumulated and analyzed, they show that the values of model parameters can be assembled and predefined boundaries could be restricted. That provoked the idea resulted in purposeful model parameters genesis (PMPG) [5] for shrinking variation boundaries of model parameters values, aiming to decrease convergence time while improve or at least save model accuracy.

The procedure for PMPG has been originally developed for singlepopulation genetic algorithm [5] and consists of six steps, as shown in the right-side of the flowchart in Figure 1. Left side of the flowchart in Figure 1 presents the working principle of standard MpGA according to $[1,6]$.

The stepwise procedure of PMPG passes through all the six steps described in Figure 1, not omitting any of them and without cycles.

\section{Results and Discussion}

In this investigation the PMPG procedure has been tested for the purposes of parameter identification of $S$. cerevisiae fed-batch cultivation when using multi-population genetic algorithm.

Experimental data of $S$. cerevisiae fed-batch cultivation is obtained in the Institute of Technical Chemistry - University of Hannover, Germany [7]. The cultivation of the yeast S. cerevisiae is performed in a 1.51 reactor, using a Schatzmann medium. Glucose in feeding solution is $50 \mathrm{~g} / \mathrm{l}$. The temperature was controlled at $30^{\circ} \mathrm{C}$, the $\mathrm{pH}$ at 5.7 . The stirrer speed was set to $500 \mathrm{rpm}$.

Considered here fed-batch cultivation of $S$. cerevisiae is characterized by keeping glucose concentration equal to or below its critical level $\left(S_{\text {crit }}=0.05 \mathrm{~g} / \mathrm{l}\right)$, sufficient dissolved oxygen $O_{2} \geq O_{2 \text { crit }}\left(O_{2 \text { crit }}\right.$ $=18 \%)$ and availability of ethanol in the broth. This state corresponds

*Corresponding author: Tania Pencheva, Institute of Biophysics and Biomedical Engineering - Bulgarian Academy of Sciences, 105 Acad. Georgi Bonchev Str., 1113 Sofia, Bulgaria, Tel: (3592) 9793648; E-mail: tania.pencheva@biomed.bas.bg

Received September 08, 2014; Accepted October 04, 2014; Published October 14,2014

Citation: Pencheva T, Angelova M (2014) Purposeful Model Parameters Genesis in Multi-population Genetic Algorithm. Global J Technol Optim 5: 164. doi: 10.4172/2229-8711.1000164

Copyright: (c) 2014 Pencheva T, et al. This is an open-access article distributed under the terms of the Creative Commons Attribution License, which permits unrestricted use, distribution, and reproduction in any medium, provided the original author and source are credited. 
Citation: Pencheva T, Angelova M (2014) Purposeful Model Parameters Genesis in Multi-population Genetic Algorithm. Global J Technol Optim 5: 164. doi: $10.4172 / 2229-8711.1000164$

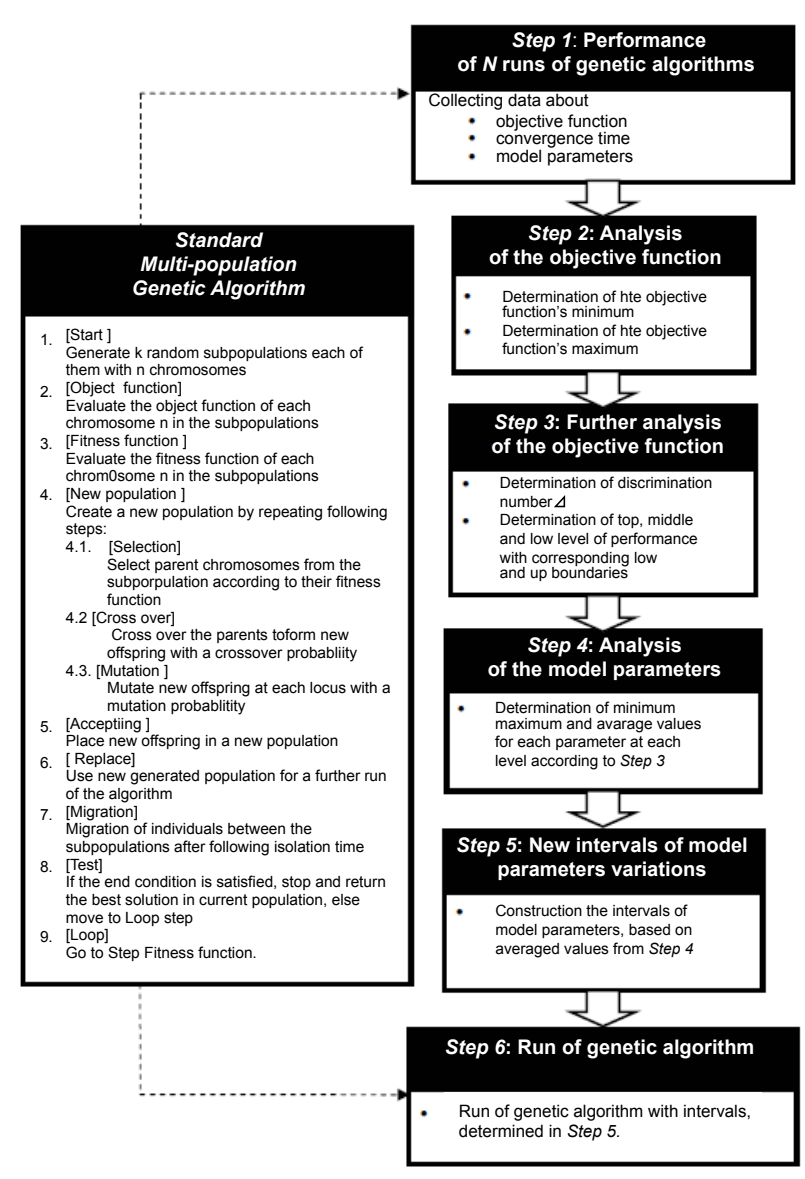

Figure 1: A flowchart of the PMPG procedure and methodology.

to the so called mixed oxidative state (FS II) according to functional state modeling approach [7]. Thus, according to the mass balance [7] and taking into account the local models for the recognized mixed oxidative state, mathematical model of $S$. cerevisiae fed-batch cultivation is presented as follows:

$$
\begin{aligned}
& \frac{d X}{d t}=\left(\mu_{2 S} \frac{S}{S+k_{S}}+\mu_{2 E} \frac{E}{E+k_{E}}\right) X-\frac{F}{V} X \\
& \frac{d S}{d t}=-\frac{\mu_{2 S}}{Y_{S X}} \frac{S}{S+k_{S}} X+\frac{F}{V}\left(S_{i n}-S\right) \\
& \frac{d E}{d t}=-\frac{\mu_{2 E}}{Y_{E X}} \frac{E}{E+k_{E}} X-\frac{F}{V} E \\
& \frac{d O_{2}}{d t}=\left(\frac{\mu_{2 E}}{Y_{E X}} \frac{E}{E+k_{E}} Y_{O E}-\frac{\mu_{2 S}}{Y_{S X}} \frac{S}{S+k_{S}} Y_{O S}\right) X+k_{L}^{O_{2}} a\left(O_{2}^{*}-O_{2}\right) \\
& \frac{d V}{d t}=F
\end{aligned}
$$

where:

$X, S, E, O_{2}$ are respectively the concentrations of biomass, $[\mathrm{g} / \mathrm{l}]$, substrate (glucose), [g/l], ethanol, [g/l], and dissolved oxygen, [\%];

$$
\mathrm{O}_{2}^{*} \text { - dissolved oxygen saturation concentration, [\%]; }
$$

$F$ - feeding rate, $[1 / \mathrm{h}]$;

$V$ - volume of bioreactor, [1];

$k_{L}^{O_{2}} a$ - volumetric oxygen transfer coefficient, [1/h];

$S_{\text {in }}$ - initial glucose concentration in the feeding solution, [g/l];

$\mu_{2 S}, \mu_{2 E}$ - maximum growth rates of substrate and ethanol, [1/h];

$k_{S}, k_{E}$ - saturation constants of substrate and ethanol, [g/l];

$Y_{i j}$ - yield coefficients, [g/g].

All functions in Eqs. (1)-(5) are continuous and differentiable and all model parameters fulfill the non-zero division requirement.

As an optimization criterion, mean square deviation between the model output and the experimental data obtained during cultivation has been used:

$$
J_{Y}=\Sigma\left(Y-Y^{*}\right)^{2} \rightarrow \min ,(6)
$$

where $Y$ is the experimental data, $Y^{*}$ is the model predicted data, $Y$ $=\left[X, S, E, O_{2}\right]$.

The developed procedure for purposeful model parameters genesis has been applied to MpGA used as a tool for parameter identification of $S$. cerevisiae fed-batch cultivation. The values of MpGA parameters and type of genetic operators are tuned according to [8]. MpGA is terminated when a certain number of generations is fulfilled, in this case 100. Scalar relative error tolerance RelTol is set to $1 \mathrm{e}^{-4}$, while the vector of absolute error tolerances (all components) AbsTol - to $1 \mathrm{e}$ ${ }^{5}$. Parameter identification of the model (1)-(5) has been performed using Genetic Algorithm Toolbox [9] in Matlab 7 environment. All the computations are performed using a PC Intel Pentium $4(2.4 \mathrm{GHz})$

\begin{tabular}{|c|c|c|c|c|c|}
\hline MpGA & \multicolumn{2}{|c|}{ Objective function } & \multicolumn{2}{|c|}{ Levels of performance } & $\begin{array}{l}\text { Average } \\
\text { convergence }\end{array}$ \\
\hline \multirow{4}{*}{ GGAP = 0.9} & \multirow{2}{*}{$\min \mathrm{J}$} & \multirow{2}{*}{0.022} & TL_LB & 0.022 & \multirow{4}{*}{159.67} \\
\hline & & & TL_UB & 0.0221 & \\
\hline & \multirow{2}{*}{$\max \mathrm{J}$} & \multirow{2}{*}{0.0222} & LL_LB & 0.0221 & \\
\hline & & & LL_UB & 0.0222 & \\
\hline \multirow{4}{*}{ GGAP $=0.8$} & \multirow{2}{*}{$\min \mathrm{J}$} & \multirow{2}{*}{0.022} & TL_LB & 0.022 & \multirow{4}{*}{151.7435} \\
\hline & & & TL_UB & 0.0221 & \\
\hline & \multirow{2}{*}{$\max \mathrm{J}$} & \multirow{2}{*}{0.0222} & LL_LB & 0.0221 & \\
\hline & & & LL_UB & 0.0222 & \\
\hline \multirow{4}{*}{ GGAP $=0.67$} & \multirow{2}{*}{$\min \mathrm{J}$} & \multirow{2}{*}{0.022} & TL_LB & 0.022 & \multirow{4}{*}{124.6432} \\
\hline & & & TL_UB & 0.022 & \\
\hline & \multirow{2}{*}{$\max \mathrm{J}$} & \multirow{2}{*}{0.0221} & LL_LB & 0.0221 & \\
\hline & & & LL_UB & 0.0221 & \\
\hline \multirow{4}{*}{ GGAP $=0.5$} & \multirow{2}{*}{$\min \mathrm{J}$} & \multirow{2}{*}{0.0221} & TL_LB & 0.0221 & \multirow{4}{*}{98.9596} \\
\hline & & & TL_UB & 0.0221 & \\
\hline & \multirow{2}{*}{$\max \mathrm{J}$} & \multirow{2}{*}{0.0222} & LL_LB & 0.0222 & \\
\hline & & & LL_UB & 0.0222 & \\
\hline
\end{tabular}
platform running Windows XP.

Following model equations (1)-(5), nine parameters for S. cerevisiae fed-batch cultivation model have been estimated altogether applying MpGA. As presented in Table 1, the algorithm has been investigated for four different values of the most sensitive towards the algorithm convergence time parameter of GA, namely generation gap (GGAP)

Table 1: Multi-population Genetic Algorithms investigations. 
[8]. For each value of GGAP, thirty runs of MpGA have been executed aiming to obtain reliable results. The results are analyzed according to achieved objective function values. For each GGAP value the minimum and the maximum of the objective function are determined, and the discrimination number is assigned according to Step 3 of the procedure [5]. After that, the algorithm proceeds with the determination of a top (TL), middle (ML) and low level (LL) of performance with corresponding low boundaries (LB) and up boundaries (UB). In the case of purposeful model parameter genesis in MpGA, all realized performances of MpGA hit only a high or a low level, i.e., without middle level. The best results for the objective function hit the interval [ $\min J ; \min J+\Delta-\varepsilon]$, while the worst solutions fall in the interval $[\min J+\Delta ; \max J]$, where $\varepsilon$ is a small number, ensuring the difference between levels.

For both recognized levels, constructed in a described way, the minimum, maximum and average values of each model parameter have been determined. Table 2 presents these values only for the top levels, according to Table 1 . The new boundaries of the model parameters are constructed in a way that the new minimum is lower but close to the minimum of the top level, and the new maximum is higher but close to the maximum of the top level. Table 3 presents previously used "wide" boundaries for each model parameter according to [10] as well as new boundaries proposed based on the procedure for purposeful model parameters genesis.

Investigated MpGA has been again applied involving newly proposed boundaries at GGAP $=0.5$. Several runs have been performed in order to obtain reliable results. Table 4 presents the average values of the objective function, computational time and model parameters before and after PMPG procedure application.

The results presented in Table 4 demonstrate the effectiveness of proposed PMPG procedure applied in MpGA. As a result of the procedure application the algorithm convergence time decreases with more than $12 \%$ and there is observed even minimal increasing of the model accuracy. Moreover, if one compares the results from MpGA with $\mathrm{GGAP}=0.9$ to these with $\mathrm{GGAP}=0.5$ obtained after the procedure of purposeful model parameters genesis, it can be seen almost two times reduction of the algorithm's convergence time without affecting the model accuracy.

Figure 2 shows results from experimental data and model prediction after PMPG procedure in MpGA, for biomass, ethanol, substrate and dissolved oxygen, respectively. The obtained results show the workability of the proposed procedure for purposeful model parameters genesis and its effectiveness when applied to MpGA.

\section{Conclusions}

In this investigation, a stepwise procedure for purposeful model parameters genesis has been tested and validated to MpGA. By shrinking the intervals of model parameters' variations, the application of the PMPG procedure improves the MpGA performance for parameter identification of a fermentation process model. As a result, MpGA becomes more than $12 \%$ faster, even with a slightly improvement of model accuracy. Moreover, if one compares the results for MpGA with $\mathrm{GGAP}=0.9$ to these with $\mathrm{GGAP}=0.5$ obtained after the PMPG procedure application, it can be seen that the algorithm convergence time reduces almost two times without affecting the model accuracy. Hence, the main advantage of the proposed procedure for PMPG is the significant improvement of the MpGA performance, expressed in decreasing of the algorithm' convergence time while saving or even meliorating the model accuracy. Such a procedure is a universal tool and can be implemented to other stochastic optimization algorithms, as well as to different objects of model parameter identification.

\begin{tabular}{|c|c|c|c|c|c|c|c|c|c|c|}
\hline MpGA & & $\mu_{2 s}$ & $\mu_{2 E}$ & $\mathbf{k}_{\mathrm{s}}$ & $\mathbf{k}_{\mathrm{E}}$ & $\mathbf{Y}_{\mathrm{sx}}$ & $\mathbf{Y}_{\mathrm{EX}}$ & $Y_{\text {os }}$ & $Y_{O E}$ & $k_{L}^{O_{2}} a$ \\
\hline \multirow{3}{*}{ GGAP $=0.9$} & $\min$ & 0.9 & 0.11 & 0.15 & 0.8 & 0.4 & 1.47 & 498.51 & 11.13 & 62.73 \\
\hline & $\max$ & 0.92 & 0.15 & 0.15 & 0.8 & 0.42 & 1.97 & 919.3 & 903.61 & 116.44 \\
\hline & aver & 0.91 & 0.13 & 0.15 & 0.8 & 0.41 & 1.69 & 659.88 & 308.79 & 83.02 \\
\hline \multirow{3}{*}{ GGAP $=0.8$} & $\min$ & 0.9 & 0.12 & 0.15 & 0.8 & 0.4 & 1.54 & 365.33 & 67.05 & 46.41 \\
\hline & $\max$ & 0.93 & 0.15 & 0.15 & 0.8 & 0.41 & 2.03 & 973.34 & 648.01 & 123 \\
\hline & aver & 0.91 & 0.13 & 0.15 & 0.8 & 0.41 & 1.73 & 716.56 & 417.4 & 90.63 \\
\hline \multirow{3}{*}{ GGAP $=0.67$} & $\min$ & 0.9 & 0.14 & 0.15 & 0.8 & 0.4 & 1.93 & 401.66 & 173.44 & 50.53 \\
\hline & $\max$ & 0.9 & 0.15 & 0.15 & 0.8 & 0.4 & 2.02 & 879.6 & 493.38 & 110.88 \\
\hline & aver & 0.9 & 0.15 & 0.15 & 0.8 & 0.4 & 1.97 & 656.6 & 228.83 & 83.15 \\
\hline \multirow{3}{*}{ GGAP $=0.5$} & $\min$ & 0.9 & 0.12 & 0.13 & 0.8 & 0.4 & 1.54 & 473.49 & 228.87 & 61.13 \\
\hline & $\max$ & 0.94 & 0.14 & 0.15 & 0.8 & 0.42 & 1.93 & 921.28 & 809.9 & 118.42 \\
\hline & aver & 0.92 & 0.13 & 0.15 & 0.8 & 0.41 & 1.72 & 656.6 & 508.5 & 92.78 \\
\hline
\end{tabular}

Table 2: Multi-population Genetic Algorithms (MpGA) values.

\begin{tabular}{|c|c|c|c|c|c|c|c|c|c|c|}
\hline MpGA & & $\mu_{2 s}$ & $\mu_{2 E}$ & $k_{s}$ & $\boldsymbol{k}_{E}$ & $Y_{s x}$ & $Y_{E X}$ & $Y_{\text {os }}$ & $Y_{O E}$ & $k_{L}^{O_{2}} a$ \\
\hline \multirow{2}{*}{$\begin{array}{l}\text { Previously } \\
\text { used boundaries }\end{array}$} & LB & 0.9 & 0.05 & 0.08 & 0.5 & 0.3 & 1 & 0.001 & 0.001 & 0.001 \\
\hline & UB & 1 & 0.15 & 0.15 & 0.8 & 10 & 10 & 1000 & 1000 & 300 \\
\hline \multirow{2}{*}{$\begin{array}{l}\text { Boundaries advisable } \\
\text { after PMPG }\end{array}$} & LB & 0.90 & 0.12 & 0.14 & 0.70 & 0.35 & 1.5 & 650 & 220 & 80 \\
\hline & UB & 0.92 & 0.15 & 0.15 & 0.80 & 0.45 & 2 & 800 & 820 & 100 \\
\hline
\end{tabular}

Table 3: Boundaries proposed based on the procedure for purposeful model parameters genesis. 
Citation: Pencheva T, Angelova M (2014) Purposeful Model Parameters Genesis in Multi-population Genetic Algorithm. Global J Technol Optim 5: 164. doi: $10.4172 / 2229-8711.1000164$

Page 4 of 4

\begin{tabular}{|c|c|c|}
\hline \multirow{2}{*}{$\begin{array}{l}\text { Parameter } / \\
\text { GGAP }=0.5\end{array}$} & \multicolumn{2}{|c|}{ MpGA } \\
\hline & Before procedure application & After procedure application \\
\hline J & 0.0221 & 0.0220 \\
\hline CPU time, $\mathrm{s}$ & 98.9596 & 86.5150 \\
\hline$\mu_{2 S}, 1 / \mathrm{h}$ & 0.91 & 0.9 \\
\hline$\mu_{2 E}, 1 / \mathrm{h}$ & 0.12 & 0.14 \\
\hline$k_{s}, g / l$ & 0.15 & 0.15 \\
\hline$k_{E}, \mathrm{~g} / \mathrm{l}$ & 0.8 & 0.8 \\
\hline$Y_{S X}, \mathrm{~g} / \mathrm{g}$ & 0.41 & 0.4 \\
\hline$Y_{E X^{\prime}} \mathrm{g} / \mathrm{g}$ & 1.62 & 1.93 \\
\hline$Y_{O S}, g / g$ & 768.61 & 696.56 \\
\hline$Y_{O E}, g / g$ & 809.90 & 291.42 \\
\hline$k_{L}^{O_{2}} a, 1 / \mathrm{h}$ & 96.34 & 88.73 \\
\hline
\end{tabular}

Table 4: The effectiveness of proposed PMPG procedure applied in MpGA (a)

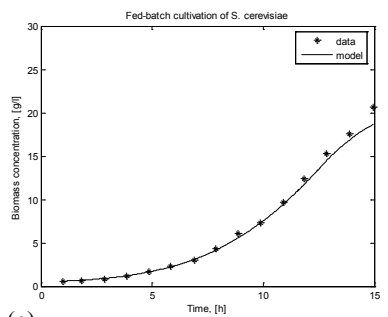

(c)

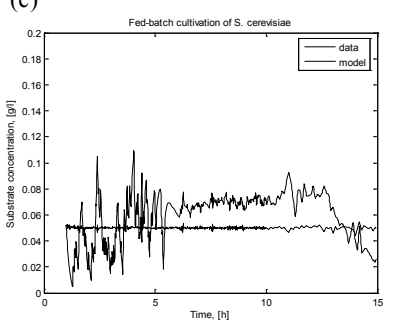

(b)

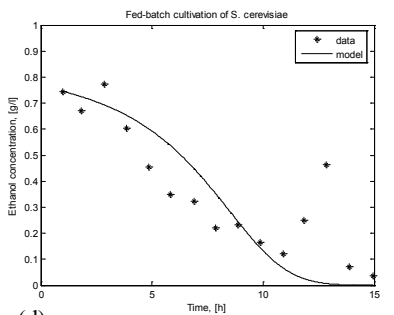

(d)

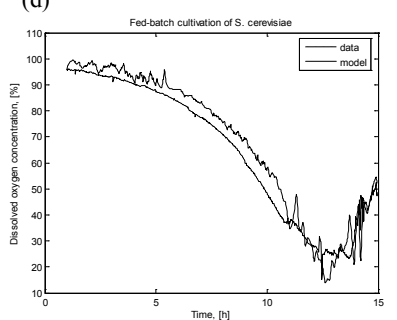

Figure 2: Model prediction compared to experimental data, (a) biomass concentration (b) ethanol concentration (c) substrate concentration and d) dissolved oxygen concentration.

\section{Acknowledgements}

This work is partially supported by National Scientific Fund of Bulgaria, Grant DMU 03-38.

\section{References}

1. Goldberg D (1989) Genetic algorithms in search, optimization and machine learning. Addison-Wiley Publishing Company, Massachusetts.

2. Adeyemo J, Enitian A (2011) Optimization of fermentation processes using evolutionary algorithms - a review. Scient Res and Ess 6(7): 1464-1472.

3. Angelova M, Tzonkov S, Pencheva T (2011) Genetic algorithms based parameter identification of yeast fed-batch cultivation. Lect Notes Comp Sci 6046: 224-231.

4. Roeva O (2006) A modified genetic algorithm for a parameter identification of fermentation processes. Biotechn \& Biotechn Equipm 20: 202-209.

5. Angelova M, Atanassov K, Pencheva T (2012) Purposeful model parameters genesis in simple genetic algorithms, Computers and Mathematics with Applications 64: 221-228.

6. Gupta D, Ghafir S (2012) An overview of methods maintaining diversity in genetic algorithms, Int. J. Emerg. Techn. and Adv Eng 2(5): 56-60.

7. Pencheva T, Roeva O, Hristozov I (2006) Functional state approach to fermentation processes modelling. Prof. Marin Drinov Academic Publishing House, Sofia.

8. Angelova M, Pencheva T (2011) Tuning genetic algorithm parameters to improve convergence time. Int. J. Chem. Eng., Article ID 646917.

9. Chipperfield AJ, Fleming P, Pohlheim H, Fonseca CM (1994) Genetic algorithm toolbox for use with MATLAB. User's guide, version 1.2. Dept of Automatic Control and System Engineering, University of Sheffield, UK.

10. Schuegerl K, Bellgardt KH (Eds.) (2000) Bioreaction engineering, modeling and control. Springer-Verlag, Berlin Heidelberg New York. 